\title{
Hybridisierung und Erotisierung des Mythos
}

Thomas Manns Roman-Tetralogie Joseph und seine Brüder

Hybridation et érotisation du mythe : la tétralogie romanesque Joseph et ses

frères de Thomas Mann

Hybridisation and Eroticization of the Myth: Thomas Mann's Novel Tetralogy

Joseph and His Brothers

\section{Thomas Pekar}

\section{(2) OpenEdition \\ Journals}

Édition électronique

URL : http://journals.openedition.org/ceg/4480

DOI : $10.4000 /$ ceg. 4480

ISSN : 2605-8359

\section{Éditeur}

Presses Universitaires de Provence

\section{Édition imprimée}

Date de publication : 2 mai 2019

Pagination : 109-120

ISBN : 979-10-320-0214-8

ISSN : 0751-4239

\section{Référence électronique}

Thomas Pekar, „Hybridisierung und Erotisierung des Mythos", Cahiers d'Études Germaniques [Online], 76 | 2019, Online erschienen am: 02 November 2011, abgerufen am 24 Januar 2021. URL: http:// journals.openedition.org/ceg/4480 ; DOI: https://doi.org/10.4000/ceg.4480 


\title{
Hybridisierung und Erotisierung des Mythos Thomas Manns Roman-Tetralogie Joseph und seine Brüder
}

\author{
Thomas PEKAR \\ Gakushuin Universität Tokyo
}

\section{Wendung zum Mythischen}

Mit seiner voluminösen Roman-Tetralogie Joseph und seine Brüder, die Thomas Mann 1926 in Deutschland zu schreiben begann (der erste Band erschien im Oktober 1933 noch im nationalsozialistischen Deutschland) und im Schweizer bzw. dann, ab 1939, im amerikanischen Exil ${ }^{1}$ fortsetzte, wo er sie 1943 auch abschloss $^{2}$, betrat er in der Weise Neuland, dass er sich, nach dem Ende seiner Beschäftigung mit der bürgerlich-ästhetischen Epoche - dieses Ende sah er 1924 mit Abschluss seines Romans Der Zauberberg für erreicht $a^{3}{ }^{3}$, nun dem Mythischen als dem Über-Individuellen und Typisch-Zeitlosen zuwandte. ${ }^{4}$ Hier soll dem Mythos-Begriff bei Thomas Mann, in Abgrenzung vor allem vom faschistischen Mythos, nachgegangen werden. ${ }^{5}$

1. Mann lebte erst in Princeton, dann, ab 1941, in Kalifornien, in Pacific Palisades.

2. Zwischen August 1936 und August 1940 hatte Thomas Mann allerdings in Hinsicht auf den Josephsroman eine Schreibpause eingelegt, um in der Zeit hauptsächlich Lotte in Weimar (1939) zu schreiben. 1943 erschien bei Bermann-Fischer in Stockholm der letzte Band der Tetralogie, betitelt Joseph, der Ernährer.

3. Vgl. Thomas Mann, Gesammelte Werke in dreizehn Bänden, Bd. XI, Frankfurt a. M., Fischer, 2. Aufl., 1974, S. 657 f. (im Folgenden als GW mit Bandangabe und Seitenangabe abgekürzt, also GW XI, S. 657 f.).

4. Kurzke sieht allerdings im Tod im Venedig (1912) ein erstmaliges Erproben des „mytische[n] Erzählen[s]“ (Hermann Kurzke, Mondwanderungen. Wegweiser durch Thomas Manns „Joseph“Romane, Frankfurt a. M., Fischer, 1993, S. 11). Dies knüpft an Dierks an, der in dieser Novelle „mythologische Anspielungen“ und im Zauberberg „eine (dünne) mythologische Bedeutungsschicht“ erkennt; der Josephsroman sei dann manifest von der „Theorie eines mythischen Bewußtseins“ und „mythischen Mustern“ bestimmt (Manfred Dierks, Studien zu Mythos und Psychologie bei Thomas Mann, Bern/ München, Francke 1972, S. 9).

5. Zum Mythos bei Thomas Mann allgemein vgl. den kurzen, aber informativen Artikel von Bastian Schlüter, „Mythos und Mythologie“, in Andreas Blödorn, Friedhelm Marx (Hrsg.), Thomas Mann Handbuch, Stuttgart, Metzler 2015, S. 253-254, auch mit Angaben zur neueren Forschungsliteratur. 
Thomas Mann konzipierte seinen Roman Joseph und seine Brüder und sein Mythos-Verständnis vor allem in einer gleichsam diametralen Opposition $\mathrm{zu}$ einem ebenfalls in dieser Zeit entwickelten folgenreichen Mythos-Konzept, welches später seinen Ausdruck in dem Hauptwerk des führenden NS-Ideologen Alfred Rosenberg fand, betitelt Der Mythus des 20. Jahrhunderts, welches zwar erst 1930 erschien, aber, wie Rosenberg selbst sagt, in seinen Grundgedanken auf 1917 zurückgehe. ${ }^{6}$ Dieses Buch gilt, neben Hitlers Mein Kampf, als das zweite Standardwerk der NS-Ideologie.

Auf Rosenberg bezieht sich Thomas Mann direkt in seinem 1942 in der Library of Congress in Washington auf Englisch gehaltenen Vortrag, dessen übersetzter Titel genau wie der Roman Joseph und seine Brüder lautet. Dieser Vortrag ist, nach Abschluss des Romans, eine Art persönlicher Rückschau Manns auf ihn. Er sagt dort: „Das Wort „Mythos“ steht ja heute in einem üblen Geruch - man braucht nur an den Titel zu denken, den der ,Philosoph“ des deutschen Faschismus, Rosenberg, der Präzeptor Hitlers, seinem bösartigen Lehrbuch beigegeben hat."7

Mann spricht hier weiter davon, dass er mit seinem Josephsroman eine „Umfunktionierung des Mythos“8 geleistet habe und führt schließlich aus: „Der Mythos wurde in diesem Buch dem Faschismus aus den Händen genommen und bis in den letzten Winkel der Sprache hinein humanisiert, - wenn die Nachwelt irgend etwas Bemerkenswertes daran finden wird, so wird es dies sein. -“9

Rosenberg ${ }^{10}$ steht in einer bestimmten Traditionslinie der Mythos-Rezeption und -Konzeption in Deutschland, welcher Thomas Mann - der zu einer Zeit, als die Beschäftigung mit dem Mythos in den Augen vieler Kritiker noch suspekt war ${ }^{11}$, Autoren wie Nietzsche, Bachofen oder Baeumler ${ }^{12}$ (bzw. den Theorien der

6. Vgl. Alfred Rosenberg, Der Mythus des 20. Jahrhunderts. Eine Wertung der seelisch-geistigen Gestaltenkämpfe unserer Zeit, München, Hoheneichen, 1939 [1930], S. 3.

7. GW XI, S. 658.

8. Diese Formel stammt von dem (neo-)marxistischen Philosophen Ernst Bloch, der sie in einem Brief an Thomas Mann vom 23. Juni 1940 prägte (vgl. Dieter Borchmeyer, „Mythos und Romanstruktur - Thomas Manns Joseph und seine ästhetischen Brüder“, in Rolf Grimminger, Iris Hermann (Hrsg.), Mythos im Text. Zur Literatur des 20. Jahrhunderts, Bielefeld, Aisthesis, 1998, S. 197).

9. GW XI, S. 658; in ähnlicher Weise schreibt Mann am 18.2.1941 an den ungarischen Religionswissenschaftler Karl Kerényi, seinen „mythologischen Berater“ (Hermann Kurzke, Thomas Mann. Das Leben als Kunstwerk, München, Beck, 1993, S. 12), dass die Psychologie „das Mittel“ sei, „den Mythos den fascistischen [sic] Dunkelmännern aus den Händen zu nehmen und ihn ins Humane ,umzufunktionieren،“” (Karl Kerényi (Hrsg.), Thomas Mann - Karl Kerényi: Gespräch in Briefen, Zürich, Rhein-Verl., 1960, S. 98).

10. Zu Rosenberg vgl. jetzt die Biografie von Volker Koop, Alfred Rosenberg. Der Wegbereiter des Holocaust. Eine Biographie, Köln/ Weimar/ Wien, Böhlau, 2016. Rosenbergs Tagebücher aus den Jahren zwischen 1936 und 1944 sind durch das United States Holocaust Memorial Museum im Internet zugänglich.

11. Vgl. einige Beispiele bei Borchmeyer, „Mythos und Romanstruktur“, S. 196.

12. Allerdings muss klar zwischen Manns grundsätzlich bejahender Bachofen- und grundsätzlich ablehnender Baeumler-Rezeption unterschieden werden, also: „Contra Baeumler, pro Bachofen“ (Elisabeth Galvan, Zur Bachofen-Rezeption in Thomas Manns „Joseph“-Roman, Frankfurt a. M., Klostermann, 1996, S. 15). 
sogenannten „konservativen Revolution“) ${ }^{13}$ reges Interesse entgegenbrachte gewiss zunächst auch nicht ferngestanden hatte. Rosenberg verband aber dann diese mythische Linie mit manifest rassistischem Gedankengut, wohingegen Thomas Mann den ganz anderen Weg der angedeuteten Humanisierung des Mythos einschlug. Den Anfang einer noch gemeinsamen mythischen Traditionslinie stellt das Begriffspaar des Apollinischen und des Dionysischen dar, welches, nachdem es von der Romantik ${ }^{14}$, insbesondere von Friedrich Creuzer, ,entdeckt ' worden war, von Friedrich Schlegel ${ }^{15}$ und Schelling aufgenommen und schließlich bei Johann Jakob Bachofen, dem Baseler Kollegen Nietzsches, entfaltet wurde, von eben Friedrich Nietzsche dann in seiner bekanntesten und bis heute geläufigen Weise in seiner Schrift Die Geburt der Tragödie aus dem Geiste der Musik (1872) ausgeführt wurde. Das, wie Nietzsche dort schreibt, „ungeheure Phänomen des Dionysischen“ steht in unmittelbarer Nachbarschaft zum „tragischen Mythus“ der Griechen. ${ }^{16}$

Nietzsche schafft mit dem Begriffspaar des Apollinischen und des Dionysischen eine folgenreiche bipolare Struktur ${ }^{17}$ (Nietzsche selbst spricht von einer „Duplicität“"18), die als grundlegend für den Mythus angesehen wird. ${ }^{19}$ Er erwartet sich insbesondere von der deutschen Musik, von dem von ihm anfänglich sehr verehrten Richard Wagner, die „Wiedergeburt des deutschen Mythus" ${ }^{20}$ und gibt dann auch eine konzise Definition und Bewertung des Mythus‘, die für viele seiner Nachfolger eine hohe Verbindlichkeit hatte; er

13. Vgl. dazu Hermann Kurzke, Thomas Mann. Epoche - Werk - Wirkung, München, Beck, 2010 [1985], S. 178-183.

14. Nachdem der italienische Philosoph Giambattista Vico die Mythenkritik der Aufklärung relativiert hatte, entstand, anfänglich bei Herder, der „,romantische Traum einer ,Neuen Mythologie““ (Manfred Frank, Der kommende Gott. Vorlesungen über die Neue Mythologie, Frankfurt a. M., Fischer, 1993, S. 122), die bei diesem auch noch mit einer „nationalen Verwurzelung der Mythologie“ (Ibid., S. 142) verbunden wurde; überhaupt sei, so Frank, „[d]ie Idee einer Neuen Mythologie [...] immer zugleich auch ein politisches Programm gewesen [...].“ (Ibid., S. 156).

15. Vgl. Schlegels Aufsatz, Über das Studium der griechischen Poesie (1797).

16. So heißt es am Anfang der Geburt der Tragödie: „Was bedeutet, gerade bei den Griechen der besten, stärksten, tapfersten Zeit, der tragische Mythus? Und das ungeheure Phänomen des Dionysischen? Was, aus ihm geboren, die Tragödie?“ (Friedrich Nietzsche, Sämtliche Werke. Kritische Studienausgabe, Bd. 1, hrsg. v. Giorgio Colli, Mazzino Montinari, München/ Berlin/ New York, Deutscher Taschenbuch Verlag/ de Gruyter, 1980 [1872], S. 12).

17. Der sich etwa die Grundkonzeption von Thomas Manns Novelle Der Tod in Venedig und wesentliche Teile seines Romans Der Zauberberg verdankt.

18. Nietzsche, Sämtliche Werke, Bd. 1, S. 25.

19. „Jeder Mythos arbeitet [...] mit einer Vielzahl von binären Codierungen [...].“ (Yves Bizeul, „Politische Mythen, Ideologien und Utopien“, in Peter Tepe/ Yves Bizeul (Hrsg)., Politische Mythen, Würzburg, Königshausen \& Neumann, 2006, S. 22.)

20. Nietzsche, Sämtliche Werke, Bd. 1, S. 147; hier wäre der Unterschied zum romantischen Projekt einer Neuen Mythologie zu sehen, die, jedenfalls bei einzelnen ihrer Protagonisten, eine übernationale „universelle Mythologie“ ist; Frank schreibt dazu: „Mehr noch und deutlicher als Novalis überschreitet [...] [Friedrich] Schlegel die nationalen und sogar die europäischen Grenzen: die neue Mythologie soll nicht nur einem Volk zur nationalen Synthesis verhelfen, sondern ,die Menschheit‘ in einem neuen Jerusalem als „Zentrum“ wiedervereinigen.“ (Manfred Frank, Der kommende Gott, S. 209). 
nennt den Mythus „das zusammengezogene Weltbild“ ${ }^{21}$ und sieht in ihm das Zeichen einer ,gesunden“ und einheitlichen Kultur: „Ohne Mythus aber geht jede Cultur ihrer gesunden schöpferischen Naturkraft verlustig: erst ein mit Mythen umstellter Horizont schliesst eine ganze Culturbewegung zur Einheit ab.“22

Diesen Zustand sieht Nietzsche allerdings in seiner Gegenwart gerade nicht als gegeben an: ,[M]an denke sich eine Cultur, die keinen festen und heiligen Ursitz hat, sondern alle Möglichkeiten zu erschöpfen und von allen Culturen sich kümmerlich zu nähren verurtheilt ist - das ist die Gegenwart, als das Resultat jenes auf Vernichtung des Mythus gerichteten Sokratismus.“ ${ }^{23}$

Die Wiedergewinnung des Mythus, der bereits von den Romantikern als verloren angesehen wurde, worauf sie mit der Forderung nach einer ,Neuen Mythologie antworteten $^{24}$ - diese Wiedergewinnung des Mythus also, den Nietzsche als Grundlage der Kultur, ja sogar als ,Fundament des Staates ansieht ${ }^{25}$, ist dann eine Aufgabe, die in seiner Nachfolge insbesondere von nationalistischen bis nationalsozialistischen Kräften in Angriff genommen wurde; und damit nähere ich mich dem an, was Thomas Mann dann als den faschistischen Mythos bezeichnet und bekämpft hat.

\section{Der faschistische Mythos und Gegenbewegungen dazu}

Die politisch rechtsorientierte, konservative, nationalkonservative, faschistische bzw. nationalsozialistische Nietzsche-Rezeption wurde noch zu Lebzeiten Nietzsches von seiner Schwester Elisabeth Förster-Nietzsche mit ihrem

21. Nietzsche, Sämtliche Werke, Bd. 1, S. 145.

22. Ibid. Dies ist in gewisser Weise als Fortsetzung von Schellings Einsichten zu verstehen, der bereits 1804 sagte: „Wo alles öffentliche Leben in die Einzelheit und Mattheit des Privatlebens zerfällt, sinkt mehr oder weniger auch die Poesie herab in diese gleichgültige Sphäre. [...] Aber eben Mythologie ist nicht in der Einzelheit möglich, kann nur aus der Totalität einer Nation, die sich als solche zugleich als Identität - als Individuum - verhält, geboren werden. [...] Eine Nation, die nichts Heiliges hat, oder der ihre Heiligthümer geraubt werden, kann auch keine wahren Tragödien haben." (Friedrich Wilhelm Joseph von Schelling, Schellings sämmtliche Werke, Abt. 1, Bd. 6, hrsg. v. Karl Friedrich August Schelling, Stuttgart/ Augsburg, Cotta 1860 [1804], S. 572f.). Frank sieht überhaupt eine Abhängigkeit Nietzsches von Schelling (vgl. Frank, Der kommende Gott, S. 344-346).

23. Nietzsche, Sämtliche Werke, Bd. 1, S. 146; unter ,Sokratismus“ versteht Nietzsche das wissenschaftlich-rationale Denken.

24. Nach Frank bezogen die Romantiker den Mythos auf die Gesellschaft: Ihre Absicht sei gewesen, „den Bestand und die Verfassung einer Gesellschaft aus einem obersten Wert zu beglaubigen“ (Frank, Der kommende Gott, S. 11) - aus dem Mythos eben.

25. „Alle Kräfte der Phantasie und des apollinischen Traumes werden erst durch den Mythus aus ihrem wahllosen Herumschweifen gerettet. Die Bilder des Mythus müssen die unbemerkt allgegenwärtigen dämonischen Wächter sein, unter deren Hut die junge Seele heranwächst, an deren Zeichen der Mann sich sein Leben und seine Kämpfe deutet: und selbst der Staat kennt keine mächtigeren ungeschriebnen Gesetze als das mythische Fundament [...].“ (Nietzsche, Sämtliche Werke, Bd. 1, S. 145). 
Nietzsche-Archiv ${ }^{26}$ und der von ihr organisierten Kompilation des Willens zur Macht (1901 erstmalig herausgegeben) angestoßen. Derjenige, der Nietzsche im Anschluss daran seit dem Ende der 1920er Jahre am stärksten als Vordenker der nationalsozialistischen Bewegung vereinnahmte, war der Philosoph und Pädagoge Alfred Baeumler ${ }^{27}$, der seit Beginn der 1930er Jahre Kontakte zu Hitler und Rosenberg hatte und später als nationalsozialistischer Ideologe im „Amt Rosenberg““, der von Alfred Rosenberg geleiteten NS-Dienststelle für Kulturpolitik und Überwachungspolitik, arbeitete. Diese Vereinnahmung bestand im Wesentlichen darin, dass Baeumler Nietzsche „eine Einheitlichkeit des Willens“ ${ }^{28}$ bzw. eine Einheitlichkeit seiner „Weltsicht“ unterschob, die er „heroischen Realismus“ ${ }^{29}$ nannte und mit dem „Germanische[n] in Nietzsche“ ${ }^{30}$ identifizierte. ${ }^{31}$

$\mathrm{Zu}$ diesem Konzept tritt dann noch bei Rosenberg der breite rassistischantisemitische Diskurs hinzu, der Ende des 19./ Anfang des 20. Jahrhunderts in Deutschland und auch Europa geführt wurde. Das vereinheitlichende Moment des Rasse-Mythus besteht in der Bildung eines homogenen Volkes bzw. seines aus, wie Rosenberg schreibt, „Lebensgefühl, staatliche[m] Stil, Kunst und Kultur“ ${ }^{32}$ bestimmten Volkstums, dem ein bestimmter „Typus“33 entsprechen soll, der die „Persönlichkeit“ bzw. den „Individualismus“ ${ }^{34}$ sowie den „Internationalismus“ bzw. den „Universalismus“ ablösen soll.

Eine besondere Reizfigur, die all diese Gegenprinzipien (also grundsätzliche Heterogenität bzw. Hybridität, Individualität, Internationalität usw.) verkörperte,

26. „Das Nietzsche-Archiv stellte sich im 20. Jahrhundert [...] zunehmend als ,Beschleuniger einer rechts-konservativen Interpretationsmaschinerie dar, die mehr und mehr in faschistisches bzw. nationalsozialistisches Fahrwasser glitt.“ (Hans-Martin Gerlach, „Politik (Faschismus, Nationalsozialismus, Sozialdemokratie, Marxismus)“, in Henning Ottmann (Hrsg.), NietzscheHandbuch. Leben - Werk - Wirkung, Stuttgart/ Weimar, Metzler, 2011, S. 500).

27. Baeumler gab eine Werkausgabe Nietzsches heraus, die ab 1930 im Kröner Verlag erschien und die sowohl eine Neuausgabe der umstrittenen (1901 erstmalig und 1906 in einer Erweiterung erschienenen) Kompilation Der Wille zur Macht. Versuch einer Umwertung aller Werte als auch eine neu von ihm erstellte Kompilation aus Nietzsches Nachlass, Die Unschuld des Werdens betitelt, umfasste. Weiter schrieb er ein Buch über Nietzsche (vgl. Alfred Baeumler, Nietzsche, der Philosoph und Politiker, Leipzig, Reclam, 2. Aufl. 1931 [1930]) und stellte einige Lebenszeugnisse vom ihm zusammen (vgl. Alfred Baeumler (Hrsg.), Nietzsche in seinen Briefen und Berichten der Zeitgenossen. Die Lebensgeschichte in Dokumenten, Leipzig, Kröner, 1932).

28. Baeumler, Nietzsche, S. 9.

29. Ibid., S. 15.

30. Ibid., S. 182.

31. In dem 1934 publizierten Aufsatz Baeumlers Nietzsche und der Nationalsozialismus wird Nietzsche eindeutig zum nordischen Vordenker der Nazi-Bewegung zurechtgestutzt: „Das männliche Zeitalter, das Zeitalter der Arbeiter und Soldaten, das von Nietzsche vorausgesagt wurde, ist im Anbrechen.“ (Alfred Baeumler, „Nietzsche und der Nationalsozialismus“, in Alfred Baeumler, Studien zur deutschen Geistesgeschichte, Berlin, Junker und Dünnhaupt, 1937, S. 293).

32. Rosenberg, Der Mythus, S. 529.

33. Der Typus entspricht der „Rassenseele“ (ibid., S. 2) bzw. der „rassengebundene[n] Volksseele“ (ibid., S. 697).

34. „Der Individualismus ist ebenso als „relativ“ erkannt wie der uferlose Universalismus.“ (ibid, S. 694). 
war für Rosenberg der Schriftsteller und Begründer der Paneuropa-Union ${ }^{35}$, Richard Nikolaus Graf Coudenhove-Kalergi, der schon durch seine österreichischjapanische Abstammung ${ }^{36}$ und seine paneuropäischen Ideen und Aktivitäten eine klare Gegenposition zu der von Rosenberg geforderten völkisch-rassisch und mythisch verbürgten Einheit darstellte. Rosenberg nennt Coudenhove-Kalergi so auch den „lauteste[n] Prediger“ eines „rasselosen Pan-Europas“.37

Dies ist deshalb erwähnenswert, weil Coudenhove-Kalergi, den Thomas Mann 1926 in Paris zum ersten Mal traf, für ihn ebenfalls zum Inbegriff eines bestimmten, allerdings positiv aufgenommenen Typus wurde - mit weitreichenden Folgen. 1926 reiste Thomas Mann mit seiner Frau Katia nach Paris; und dies war genau die Zeit, in der er seinen Josephsroman zu konzipieren und zu schreiben begann. ${ }^{38}$ Über seinen Aufenthalt dort vom 20. bis zum 29. Januar hat er in seinem Essay Pariser Rechenschaft berichtet. ${ }^{39}$ Er reiste nach Paris, um dort die „Völkerversöhnung [...] zu befördern“" ${ }^{40}$; bei den vielen Veranstaltungen, an denen er dort teilnahm, traf er auch Coudenhove-Kalergi und beschreibt ihn so:

Coudenhove [...] ist einer der merkwürdigsten und übrigens schönsten Menschen, die mir vorgekommen. Zur Hälfte Japaner, zur anderen Hälfte gemischt aus dem internationalen Adelsgeblüt Europas, wie man weiß, stellt er wirklich einen eurasischen Typus vornehmer Weltmenschlichkeit dar, der außerordentlich fesselt und vor welcher der Durchschnittsdeutsche sich recht provinzlerisch fühlt. Zwei Falten zwischen den fernöstlich sitzenden schwarzen Augen, unter der reinen, festen und stolz getragenen Stirn, verleihen seinem Lächeln etwas Ernstes und Entschlossenes. ${ }^{41}$

\section{Zur Hybridität von Thomas Manns Josephsroman}

Thomas Mann nimmt also Coudenhove als einen faszinierenden eurasischen Mischtypus auf, der ihm „Weltmenschlichkeit“ repräsentiert. Wenn man dies in den Kontext seines Josephsroman stellt, auf den im Paris-Essay zahlreiche

35. Diese Union wurde 1922 gegründet und ist die älteste gegenwärtig noch bestehende europäische Einigungsbewegung, die auf die Vereinigung Europas als einer christlich-konservativen Wertegemeinschaft abzielt.

36. Sein Vater, Heinrich von Coudenhove, war österreichisch-ungarischer Geschäftsträger in Japan, seine Mutter, Mitsuko Coudenhove-Kalergi, Japanerin. 1895 ging sie, zusammen mit ihrem Mann, nach Österreich (als eine der ersten Japanerinnen überhaupt), wo sie, nach seinem frühen Tod, die Verwaltung der Familiengüter in Westböhmen übernahm und sich um die Erziehung ihrer sieben Kinder kümmerte. Später zog sie nach Wien bzw. Mödling bei Wien, wo sie 1941 verstarb.

37. Rosenberg, Mythus, S. 639.

38. Eine erste Erwähnung des Josephsromans ist in einem Brief Manns an Ernst Bertram am 4.2.1925 zu finden (vgl. Inge Jens (Hrsg.), Thomas Mann an Ernst Bertram. Briefe aus den Jahren 1910-1955, Pfullingen, Neske, 1960, S. 136 und Thomas Mann, Große kommentierte Frankfurter Ausgabe, Bd. 15.2, Frankfurt a. M., Fischer, 2001 ff., S. 825 (im Folgenden als GKFA mit Bandangabe und Seitenangabe abgekürzt, also GKFA 15.2, S. 825)).

39. GKFA 15.1, S. 1115-1214.

40. So wird diese Reise im Kommentar bewertet (vgl. GKFA 15.2, S. 781).

41. GKFA 15.1, S. 1157. 
Anspielungen hindeuten ${ }^{42}$, so liegt die Überlegung nahe, dass Thomas Mann vielleicht sogar von diesem repräsentativen ,Mischtypus zu seinem in und durch den Josephsroman gestalteten ,Mischmythus' bzw. zu seinem dem faschistischen Mythos diametral entgegengesetzten hybriden Mythos-Verständnis gekommen sein $\mathrm{mag}^{43}$, gespeist letztendlich von einer erotischen Faszination. Das monolithische Gebilde eines überindividuellen Mythos (wie in faschistischen Vorstellungen von einer Rasse, einem Volk und einem Führer) wird nicht nur durch die Vielheit der Mythen, sondern auch durch ihre Anwendung auf einzelne Menschen (bzw. Figuren) zersetzt - dies benennt das Stichwort von der ,Psychologisierung“, die zugleich eine ,Erotisierung“ bedeutet, da individuellpsychologische Konflikte im Josephsroman sich in erster Linie im erotischen Bereich entzünden. ${ }^{44}$

Zwischen dieser Mann'schen Beschreibung Coudenhoves und wesentlichen Zügen der Hauptfigur seines Josephsroman gibt es zahlreiche Überschneidungen, nämlich vor allem in Hinsicht auf ,Schönheit', wobei der ja durchaus auch oder sogar primär homoerotisch empfindende Thomas Mann nicht zwischen männlicher und weiblicher Schönheit unterschied ${ }^{45}$, ja eigentlich männliche, besonders jünglingshaft-männliche Schönheit präferierte: Dies wird im Roman bei der Beschreibung Josephs überdeutlich, der ein eigenes Kapitel, „Von der Schönheit“ betitelt, gewidmet ist; hier wird Joseph als androgyne ${ }^{46}$, also männlich-weibliche (in heutiger Terminologie: transgender oder genderqueer bzw. non-binary/nicht-binäre) Schönheit gefasst. ${ }^{47}$

42. In Anspielung auf seine Mittelmeerreise 1925, die ihn von Venedig nach Ägypten geführt hatte (vgl. GKFA 15.2, S. 825), schreibt er, dass „das Ägyptische“ in seiner Literatur „,noch nie zum Vorschein gekommen“ sei - und fügt dann kryptisch hinzu: „Es wird schon.“ (GKFA 15.1, S. 1166). Wenig später trifft er einen französischen Diplomaten, mit dem er über Ägypten redet. Er prahlt hier mit seinen ägyptologischen Kenntnissen und gibt einen direkten Einblick in seine Romanwerkstatt, wenn er den von ihm erfundenen und dann in seinem Roman benutzten Namen für Potiphars Frau (die in der biblischen Josefs-Geschichte namenlos bleibt) verrät.

43. Wohingegen Rosenberg den umgekehrten Weg gegangen ist - vom totalitären Mythus zum homogenen arischen (Volks-)Typus.

44. Das Musterbeispiel dafür ist die auf Joseph gerichtete tragisch-unerfüllte Liebessehnsucht von Potiphars Frau.

45. Im Roman verschmelzen in dieser Hinsicht auf Schönheit Mutter (Rahel) und Sohn (Joseph): Beide sind „hübsch und schön“ (GW IV, S. 10 und S. 228). Jaakob liebt Joseph auch deshalb besonders, weil er in ihm seine geliebte Frau Rahel erkennt. Sowohl die Sitte der Beschneidung wie eben auch das Bewusstsein, hübsch und schön zu sein, erzeugen bei Joseph „ein gewisses weibliches Bewußtsein“ (GW IV, S. 80). Bereits in der Bibel wird auf die Schönheit Josephs hingewiesen (vgl. Gen 39, 6).

46. Diese Androgynität wird auf mythologischer Ebene durch Josephs besondere Beziehung zum Mond, der „in seiner Mittel- und Mittlerstellung zwischen der solaren und der irdischen, der geistigen und der stofflichen Welt“ (Kurzke, Mondwanderungen, S. 20) ein androgynes Symbol ist, hergestellt.

47. „Joseph war siebzehn Jahre alt und in den Augen aller, die ihn sahen, der Schönste unter den Menschenkindern. [...] Mit siebzehn, das ist wahr, kann einer schöner sein als Weib und Mann, schön wie Weib und Mann, schön von beiden Seiten her und auf alle Weise, hübsch und schön, $\mathrm{da} \beta$ es zum Gaffen und Sichvergaffen ist für Weib und Mann. So war es mit Rahels Sohn [= Joseph], und darum heißt es, daß er der Schönste war unter den Menschenkindern.“ (GW IV, S. 393 und S. 395). 
Sicher gehen in die Josephs-Figur auch verschiedene weitere Personen aus Thomas Manns Umgebung ein, so z. B. auch seine Ehefrau Katia, deren oft gerühmte große, schwarze Augen sich dann auch bei Joseph wiederfinden (bzw. erst bei seiner Mutter, Rahel).

Man kann also sagen, dass sich Thomas Mann in dieser Zeit der Konzeption und ersten Schreibanfänge des Josephsromans ein Mythos-Verständnis erarbeitete, welches mit diesem gedanklich und erotisch $^{48}$ favorisierten ,Mischtypus‘, repräsentativ für ,Weltmenschlichkeit‘, verbunden war und diesen Typus fundieren und legitimieren sollte.

\section{Die fatale deutsche Mythos-Tradition}

Diese Frage der hybriden Mythos-Konzeption wird in Thomas Manns Paris-Essay weiter in der Weise aufgenommen, dass er seine Coudenhove-Beschreibung mit seiner Diskussion einer spezifisch deutschen Denkweise ${ }^{49}$ bzw. MythosTradition umrahmt, von der er sich explizit abgrenzt. In dieser Abgrenzung finden sich wesentliche Elemente seines neuen, dem entgegengesetzten MythosVerständnisses, für welches ihm Coudenhove gewissermaßen schon Bild und Figur war. Wovon grenzt Thomas Mann sich ab? Es ist diejenige MythusTradition, die, so sagt er hier, in Baeumlers Einleitung zu Bachofen zu finden ist, die unter dem Titel Bachofen, der Mythologe der Romantik ${ }^{50}$ gerade auch in diesem Jahr 1926 erschienen war. Von ihr führt ein direkter Weg zu Rosenbergs Mythus-Konzeption ${ }^{51}$; bei Baeumler handelt es sich um einen bzw. den NaziMythos (avant la lettre), um einen totalitären Mythos also - und dies sieht

48. Die erotische Dimension der Beziehung zu Coudenhove wird im Paris-Essay ebenfalls angegeben: Thomas Mann schildert noch eine weitere Begegnung, in einem Pariser Theater, wo er sich mit ihm in den Pausen unterhält und dieser ihm „das Geständnis einer starken Neigung“ (schon diese Wortwahl hat eine gewisse Doppeldeutigkeit) für Manns 1915 erschienenen Aufsatz über Friedrich den Großen (Friedrich und die große Koalition; GKFA 15.1, S. 55-122) macht; weiter bekennt sich Coudenhove hier zu seiner heroischen Ethik, die er „als Japaner“ (GKFA 15.1, S. 1163) habe. Beide Hinweise, sowohl auf den Preußenkönig als auch auf die japanische Kriegsethik (Bushidō), haben zweifellos einen „homoerotischen Subtext“ (Heinrich Detering, „Nachwort und Dank“, in GKFA 14.2, S. 591). Dass der Männlichkeitswahn der Samurai-Ethik des Bushidō homoerotisch bzw. manifest homosexuell fundiert war, ist in Japan allgemein bekannt. Und was den Preußenkönig betrifft: Vielen Historikern gilt Friedrich der Große als homosexuell.

49. Er spricht zunächst von der deutschen „Neigung zu den Mächten des Unbewußten und des vorkosmisch-lebensträchtigen Dunkels“ und der „Tendenz zum Abgrunde, zur Unform und zum Chaos“, was die Deutschen „zu rechten Sorgenkindern des Lebens mache.“ (GKFA 15.1, S. 1127).

50. Vgl. Alfred Baeumler, „Einleitung. Bachofen der Mythologe der Romantik“, in Manfred Schroeter (Hrsg.), Der Mythus von Orient und Occident. Eine Metaphysik der Alten Welt. Aus den Werken von Bachofen, München, Beck, 1926, S. XXIII-CCXCIV.

51. Diese Mythos-Konzeption diente also dazu, die faschistische Ideologie bei der Bevölkerung zu verankern; vgl. zu diesem Zusammenhang von Mythos und Ideologie: Yves Bizeul, „Theorien der politischen Mythen und Rituale“, in Yves Bizeul (Hrsg.), Politische Mythen und Rituale in Deutschland, Frankreich und Polen, Berlin, Dunker und Humblot, 2000, S. 18. 
Thomas Mann im Paris-Essay mit bewunderungswürdiger Klarheit ${ }^{52}$ (er spricht auch direkt von der Gefahr „des Faschismus“53): Er zeichnet hier den (Irr-)Weg der von Baeumler skizzierten und in seine irrationale Denkweise mündenden romantischen Tradition nach, vor der er eindringlich warnt und zu der er seine Gegenposition entwirft.

\section{Thomas Manns Gegenposition zum faschistischen Mythos}

Wie diese Gegenposition, die des von erotischer Energie gespeisten hybriden Mythos, im Josephsroman genauer ausgestaltet ist, soll jetzt abschließend dargestellt werden.

Eine Auflistung und Analyse all der Mythen, die Thomas Mann in seinem Josephsroman verwendet, würde den Rahmen dieses Aufsatzes bei weitem sprengen und wäre auch überflüssig, da man dies in der Forschungsliteratur schon ausführlich getan hat. ${ }^{54}$ Die wichtigsten Mythen, die Thomas Mann miteinander vermischt, sind jüdische, christliche, ägyptische, babylonisch-kanaanitische und hellenistische. ${ }^{55}$ Dabei wird jedoch oft eine Mythen-Hybridisierung ${ }^{56}$ übersehen, nämlich die Mischung dieser genannten ,alten“ Mythen ${ }^{57}$ mit dem neuzeitlichen

52. Zu optimistisch war allerdings seine Einschätzung von der Unumkehrbarkeit des demokratischen Weges für Deutschland, die er einige Male betont, z. B.: „Der Weg ins Vordemokratische zurück ist jedoch ungangbar." (GKFA 15.1, S. 1158).

53. GKFA 15.1, S. 1162. „Thomas Mann verwendet den Begriff ,Faschismus‘ für eine internationale Erscheinung im Europa der zwanziger und dreißiger Jahre. Er betrachtet den deutschen Nationalismus als eine Spielart dieses internationalen Faschismus.“ (Kurze, Thomas Mann, S. 230); vgl. dazu auch Lothar Pikulik, Thomas Mann und der Faschismus: Wahrnehmung - Erkenntnisinteresse - Widerstand, Hildesheim, Olms, 2013.

54. Vgl. vor allem Kurzke, Mondwanderungen, Bernd-Jürgen Fischer, Handbuch zu Thomas Manns „Josephsromanen“, Tübingen/ Basel, Francke, 2002 und Jan Assmann, Thomas Mann und Ägypten: Mythos und Monotheismus in den Josephsromanen, München, Beck 2006. Die JosephsFigur selbst ist ein gutes Beispiel für die Hybridisierung, die Kurzke so angibt: „Joseph wird von den Ägyptern ,Adôn“ genannt, denn er ist der schöne griechische Jüngling Adonis [...]. Er ist der babylonische Tammuz, der Zerrissene und Geopferte, ,der Usiri des Ostens`, der wie Christus den Tod besiegt, und Dionysos Zagreus, der als Knabe von den Titanen zerstückelt wurde. Er ist Gilgamesch [...]. Er ist - als Osarsiph - der ägyptische Osiris [...]. Joseph ist Ödipus vor der Sphinx, und Joseph ist Christus [...].“ (Kurzke, Mondwanderungen, S. 67-69).

55. Vgl. ibid., S. 95; Kurzke spricht hier von einer „synkretistische[n] Mythenkombinatorik“.

56. Der literaturwissenschaftliche Begriff der ,Hybridisierung“ (bzw. der ,hybriden Konstruktion“) geht auf den russischen Literaturwissenschaftler Michail Bachtin zurück. (vgl. Michail M. Bachtin, Die Ästhetik des Wortes, hrsg. v. Rainer Grübel, Frankfurt a. M., Suhrkamp, 1979, S. 195). Um Thomas Manns Verfahren der Mythen-Mischung zu charakterisieren, werden verschiedene Begriffe benutzt, deren Bedeutungsgehalt jedoch ähnlich ist; vgl. z.B.: „Grundsätzlich gilt, dass Thomas Mann die überlieferte Geschichte von Joseph und seinen Brüdern auf kaum erschöpfend zu rekonstruierende Weise mit Mythen diverser Kulturkreise amalgamiert.“ (Thorsten Wilhelmy, Legitimitätsstrategien der Mythosrezeption, Würzburg, Königshausen \& Neumann, 2004, S. 84).

57. Mann spricht davon, dass in seinem Roman „die Mythologien aller Welt, die ebräische, babylonische, ägyptische, griechische so bunt durcheinander“ gehen würden, „daß man sich kaum noch darauf besinnen wird, ein biblisch-jüdisches Geschichtenbuch vor sich zu haben.“ (GW XI, S. 664). 
Mythos ,Amerika' (dem American Dream) ${ }^{58}$, was sich besonders im vierten und letzten Band des Josephsromans, Joseph, der Ernährer betitelt, zeigt.

In seiner Selbstauslegung des Romans lässt Thomas Mann Josephs Ich einmünden „,ins Kollektive, Gemeinsame“: „[D]er Gegensatz [...] von Individuum und Kollektiv“ hebe sich „in der Demokratie der Zukunft“ auf - und diese lebe von dem „amerikanischen Mythos“; oder, besser gesagt, ist dieser amerikanische Mythos selbst, ist „freedom and democracy“ entsprechend dem „American way of life“..$^{59}$

Thomas Mann hat zweifellos in diesen 1940er Jahren an den American Dream geglaubt; er selbst spricht, am Ende seines 1942 in der Washingtoner Library of Congress gehaltenen Vortrags, dessen englischer Original-Titel The Theme of the Joseph Novels ${ }^{60}$ lautet, gleich zweimal vom „American myth“ und weiter davon, dass sein Roman „in contact“61 mit diesem Mythos vollendet worden sei (diese Passage fehlt im Übrigen in der deutschen Übersetzung dieses Vortrags in Manns Gesammelten Werken). ${ }^{62}$

Diesen American Myth sah Thomas Mann vor allem in dem amerikanischen Präsidenten Franklin D. Roosevelt und seinem New Deal verkörpert; zu Roosevelt hatte Thomas Mann eine enge, auch durch persönliche Kontakte vertiefte Beziehung. ${ }^{63}$ Die von Roosevelt 1933 gleich nach seinem Amtsantritt in Gang gesetzten wirtschaftlichen und sozialen Reformen (u. a. Regulierung der Finanzmärkte und Einführung von Sozialversicherungen), die unter den Namen New Deal bekannt wurden, setzt Thomas Mann in den Maßnahmen Josephs in Ägypten (den er dort u. a. die Bewässerungsverhältnisse verbessern und gar eine Umverteilung des Reichtums in Angriff nehmen lässt) ${ }^{64}$ ein literarisches Denkmal. ${ }^{65}$

58. Vgl. dazu aus der Perspektive eines ,modernen Mythos“ Sebastian Jobs, „American Dream“, in Stephanie Wodianka, Juliane Ebert (Hrsg.), Metzler Lexikon moderner Mythen. Figuren, Konzepte, Ereignisse, Stuttgart/ Weimar, Metzler, 2014, S. 18-21. Man könnte diesen „American Dream“ natürlich selbst schon als ein hybrides oder auch synkretisches Produkt ansehen.

59. GW XI, S. 667.

60. Vgl. Thomas Mann, The Theme of the Joseph Novels, Washington, U.S. Government Printing Office, 1942.

61. Ibid., S. 23.

62. Vgl. GW XI, S. 654-669; Detering weist auf diese Auslassung hin (vgl. Heinrich Detering, Thomas Manns amerikanische Religion. Theologie, Politik und Literatur im kalifornischen Exil, Frankfurt a. M., Fischer, 2012, S. 122 und S. 248, Anm. 266).

63. Vgl. Hans Rudolf Vaget, Thomas Mann, der Amerikaner. Leben und Werk im amerikanischen Exil, Frankfurt a. M., Fischer, 2. Aufl., 2012.

64. Vgl. dazu ibid., S. 152 und die abschließende Aussage: „Somit darf das Regime Josephs, des Ernährers, in dem Sinne als eine spielerische Bezugnahme auf Roosevelt und den New Deal aufgefasst werden [...].“ (Ibid., S. 155).

65. Mann sagt selbst, dass sich der „New Deal [...] in Josephs magischer Wirtschaftsadministration unverkennbar widerspiegelt.“ (GW XI, S. 680) Und Joseph, der Ernährer, trägt auch idealisierte Züge von Roosevelt selbst. Detering spricht davon, dass Roosevelts „idealisiertes Bild die Darstellung Josephs des Ernährers [...] mitbestimmt [...].“ (Detering, Thomas Manns amerikanische Religion, S. 122). 
Es ist wichtig zu sehen, dass Thomas Mann diesen amerikanischen „Way of Life“" ${ }^{66}$, den er in Roosevelt, im New Deal und dann letztendlich auch im amerikanischen Kriegseintritt, den er als Kampf für weltweite Freiheit, Demokratie und Humanismus begriff, als Mythos fasste, als, wie er sagt, „the American myth, which is alive today." 67

Dies zeigt Thomas Manns Einsicht in die Notwendigkeit der Mythenerzeugung auch im 20. Jahrhundert - er kontert die Mythoskonzeption, Mythenerfindung und Mythenbemächtigungsversuche ${ }^{68}$ der faschistischen ,Dunkelmänner durch die Schaffung eines anderen Narrativs, eines vielschichtigen, Vergangenheit und Gegenwart umgreifenden humanen Gegenmythos, seines Josephsromans. ${ }^{69}$ Und dies erscheint mir genau das zu sein, was Roland Barthes, der vielleicht immer noch scharfsinnigste Mythologe unserer Zeit, auch empfiehlt: „À vrai dire, la meilleure arme contre le mythe, c'est peut-être de le mythifier à son tour, c'est de produire un mythe artificiel: et ce mythe reconstitué sera une véritable mythologie." 70

Als einen solchen konstruierten bzw. artifiziellen Mythos - als Waffe im Kampf gegen den homogen-faschistischen Mythos von Volk und Rasse - sehe ich Thomas Manns Josephsroman an, der damit eine solche wahre und wahrhaftige Mythologie, die Mythologie der ,Weltmenschlichkeit‘ wäre. ${ }^{71}$

66. „,Y]ou, too, live in a tradition here, walk in footsteps, in paternal footsteps, which you call your „Way of Life.““” (Mann, The Theme, S. 23).

67. Ibid., S. 23. Detering spricht in dieser Hinsicht davon, dass Mann „einen Menschheits-Mythos“ entwerfe, „der ein Kontinuum umfasst vom alten Israel bis zur Neuen Welt.“ (Detering, Thomas Manns amerikanische Religion, S. 186).

68. Auf die Walter Benjamin 1939 mit diesem Satz hinweist: „[N]ichts Geringeres schwebt den Faschisten vor, als des Mythos sich zu bemächtigen." (Walter Benjamin, Gesammelte Schriften II.2, hrsg. von Rolf Tiedemann/ Hermann Schweppenhäuser, Frankfurt a. M., Suhrkamp, 2. Aufl., 1977, S. 582).

69. Damit grenzt er sich von einer naiv-aufklärerischen Position ab, die glaubt, durch den Appell an die Vernunft allein Mythen und Irrationalität überwinden zu können. Eine solche Meinung vertrat z.B. der französische Aufklärer Fontenelle im 18. Jahrhundert, der behauptete, dass uns die Mythen „die Geschichte der Irrtümer des menschlichen Geistes“ liefern würden, die „der vernünftigen Überprüfung nicht standhalten [...] können." (Zit. nach Frank, Der kommende Gott, S. 116).

70. Roland Barthes, Mythologies, Paris, Seuil, 1970, S. 209.

71. Sinnvoll wäre es, Teile von Manns Josephsroman als einen politischen Mythos zu lesen, da er u. a. den Ursprung einer politischen Ära, des New Deals, und die Geschichte einer herausragenden Persönlichkeit, nämlich Roosevelts, erzählt; zu diesem Verständnis des politischen Mythos‘vgl. Bizeul, Theorie der politischen Mythen, S. 17. 
